Perspective

\title{
High Entropy Alloys: Ready to Set Sail?
}

\author{
Indranil Basu $1,2, *(1)$ and Jeff Th. M. De Hosson $1, *$ (1) \\ 1 Department of Applied Physics, Zernike Institute for Advanced Materials, University of Groningen, 9747AG \\ Groningen, The Netherlands \\ 2 Laboratory of Metal Physics and Technology, Department of Materials, ETH Zurich, 8093 Zurich, Switzerland \\ * Correspondence: ibasu@ethz.ch (I.B.); j.t.m.de.hosson@rug.nl (J.T.M.D.H.)
}

Received: 24 December 2019; Accepted: 27 January 2020; Published: 29 January 2020

\begin{abstract}
Over the past decade, high entropy alloys (HEAs) have transcended the frontiers of material development in terms of their unprecedented structural and functional properties compared to their counterpart conventional alloys. The possibility to explore a vast compositional space further renders this area of research extremely promising in the near future for discovering society-changing materials. The introduction of HEAs has also brought forth a paradigm shift in the existing knowledge about material design and development. It is in this regard that a fundamental understanding of the metal physics of these alloys is critical in propelling mechanism-based HEA design. The current paper highlights some of the critical viewpoints that need greater attention in the future with respect to designing mechanically and functionally advanced materials. In particular, the interplay of large compositional gradients and defect topologies in these alloys and their corresponding impact on overall mechanical response are highlighted. From the point of view of functional response, such chemistry vis-à-vis topology correlations are extended to novel class of nano-porous HEAs that beat thermal coarsening effects despite a high surface to volume ratio owing to retarded diffusion kinetics. Recommendations on material design with regards to their potential use in diverse applications such as energy storage, actuators, and as piezoelectrics are additionally considered.
\end{abstract}

Keywords: serrated flow; thermal coarsening; actuators; phase transformation; nanoporous metals and alloys

\section{Introduction}

The classical rationale behind complex concentrated alloys (CCAs), more popularly referred to as high entropy alloys, comprises the addition of four or five elements in equiatomic and near-equiatomic proportions that eventually generate a single-phase solid solution $[1,2]$. The theoretical feasibility of such a counterintuitive alloy design stems from the concept of entropic stabilization, wherein a large number of elements would raise the configurational entropy and simultaneously overcome the enthalpies associated with the formation of intermetallic compounds. Owing to the inherent complexity of entropic stabilization, such multicomponent alloys have been observed to display peculiar characteristics that were summarized by Yeh and co-workers [1] as the four core effects: (i) The maximization of configurational entropy, owing to which the term high entropy alloys (HEAs) was coined; (ii) lattice strain due to large variation in atomic sizes of constituent elements; (iii) sluggish diffusion kinetics due to frustrated crystal structures; and iv) unusual properties displayed due to diverse interatomic interactions, also referred to as the "cocktail" effect [3].

However, the universality of occurrence of the abovementioned core effects in HEAs is debatable [4-9]. For instance, the very theory describing entropic stabilization has found little experimental validation, indicated by the fact that majority of HEAs that have fabricated till now either exist as multiphase alloys or decompose to more than one phase at thermodynamic 
equilibrium [6-8,10-12]. This is primarily because of the fact that the postulation by Yeh et al. on the maximization of configurational entropy remains valid at melting temperatures [13]. On the other hand, most experimental alloys are characterized at room temperatures, which renders significant microstructural and phase reordering driven by local stress/strain distributions, local compositional fluctuations, and interatomic interactions among constituent elements under diverse thermomechanical processing schemes $[13,14]$. Nevertheless, due to the widespread popularity of the postulated core effects within the community, they are still being actively associated with HEAs.

While the search for stable single-phase HEAs via combinatorial approaches continues $[9,15]$, a large part of the research in the field delves into exploiting multiphase HEAs as means to design materials with significantly enhanced structural and functional properties $[10,13]$. Of special note in this regard are the less stringent alloying routes that provide access to a much larger compositional space in comparison with conventional alloys. This facilitates a mechanism driven compositional tuning of alloys, wherein the high solid solution content of individual elements can be tailored in order to generate unique features, such as spinodal structures [16], nano-scale coherent precipitates [17], and the modification of stacking fault energies $[18,19]$, that give rise to interesting metal physics at intrinsic length scales. The advantages of this are clearly visible from the wide-spread future applications proposed for HEAs with unprecedented combinations of structural and functional properties [13,20-23].

In light of the aforementioned aspects of HEAs, it has become imperative to understand the independent and interdependent effects of the spatial distribution of linear and planar defects (i.e., defect topology) and compositional fluctuations existing in these alloys on the local mechanical and functional responses.

\section{Crystallographic Defects versus Compositional Variation: A Tale of Two Effects}

A critical aspect that influences mechanical responses in materials is the dependence of defect topologies and compositional gradients upon length scale dynamics. When considering crystal defects, the particular game players with respect to local plasticity are line defects and surface/interface defects, as well as their mutual interactions. Adding to this, the effect of local compositional fluctuations that could be considerable in HEAs further adds another layer of complexity in terms of local stress evolution and corresponding strain accommodation. Owing to such compositional diversity, metal physics in HEAs therefore offers an interesting playground wherein a superposition of multiple strengthening mechanisms that can interact and augment each other's contribution takes place. It must be understood that even though the observed metal physics in HEAs are derived from those that are seen for conventional alloys and superalloys, the compositional flexibility and simultaneous activation of different strengthening modes in the former potentially places them as more suitable candidates for mechanism-based alloy design.

The influence of local chemistry on meso-scale microstructures serves as a key design aspect in HEAs in regard to tailoring alloys with significantly improved structural responses. Owing to the absence of a primary solvent, local elemental partitioning in these alloys often results in the formation of phase interfaces. Depending upon whether such interfaces are crystallographically similar or dissimilar, the corresponding impact on the plasticity and strengthening behavior is distinct. For instance, one of the commonly studied systems in this regard is the $\mathrm{Al}_{x} \mathrm{CoCrFeNi}$, wherein increasing the $\mathrm{Al}$ content drives a crystallographic transition from an Face-centered cubic (FCC) solid solution to a Body-centered cubic (BCC) phase [1]. Moreover, the BCC phases that exist in this alloy are further known to spinodally decompose into ordered B2 (enriched with $\mathrm{Al}$ and $\mathrm{Ni}$ ) and disordered BCC structures [16,23-25]. It must be highlighted that the underlying strength contribution and metal physics are entirely different when considering such different interface types, and these become critical to appraise when engineering HEAs with strengthening across different length scales. 


\subsection{Defect Generation and Strengthening Behavior across Crystallographically Similar Interfaces}

In a recent study [16], the nano-indentation response of $\mathrm{BCC}$ grains in $\mathrm{Al}_{0.7} \mathrm{CoCrFeNi}$ alloys indicated series of random displacement bursts. The observed pop-ins showed varying amplitudes and seemed to be more obvious at lower indentation loads and smaller penetration depths (Figure 1c). Serrated flow characteristics are the fingerprint of jerky dislocation kinetics, as these arise from intermittent intervals of obstructed dislocation motion. In the present case, it was shown that the spinodally decomposed ordered B2 and disordered A2 phases generated interfaces that gave rise to simultaneous spinodal hardening and order-hardening effects (Figure 1a,b). These effects typically manifest at deformation length scales that are comparable to the mean size of the A2 phase, that is 100-200 nm (Figure 1d,e). Considering the fact that spinodal strengthening in BCC crystal structures can be significantly larger than FCC spinodal alloys due to the sizable contribution of both elastic coherency strains and hardening from the modulus differential in the former, it has been shown that the strengthening potential in spinodal BCC HEAs can be as high as $0.5 \mathrm{GPa}$. In another study [17], it was shown that the addition of $\mathrm{Ti}$ and $\mathrm{Al}$ to single phase FCC CoCrFeNi HEAs leads to precipitation hardening effects due to presence of ordered FCC precipitates in a random FCC matrix, and these contribute to a strengthening increment between 0.3 and $0.4 \mathrm{GPa}$, which is significantly larger than counterpart contributions from strain hardening, grain boundary hardening, and solid solution strengthening. Lately, the concept of utilizing such spinodally-induced strengthening and order hardening effects in HEAs has given rise to a new generation of modulated, nano-phase structured, BCC-refractory HEAs that mimic super alloy type microstructures [26,27].

a)

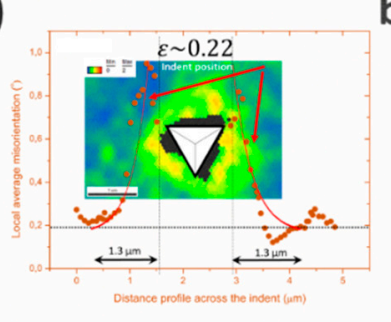

d)

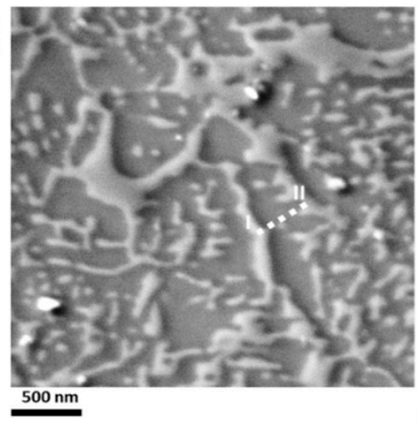

b)
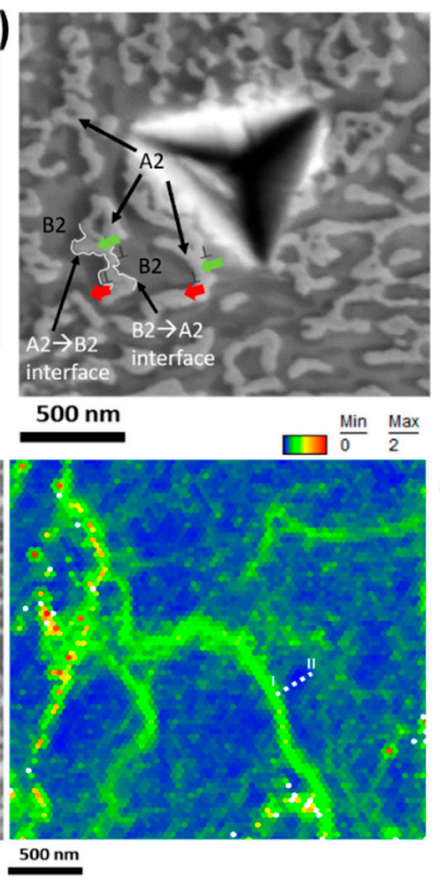

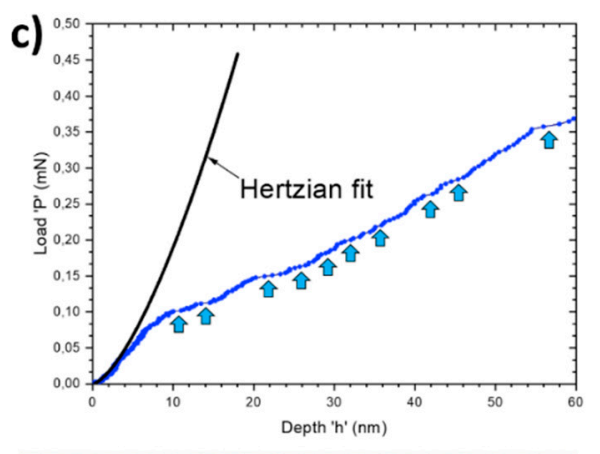

e)

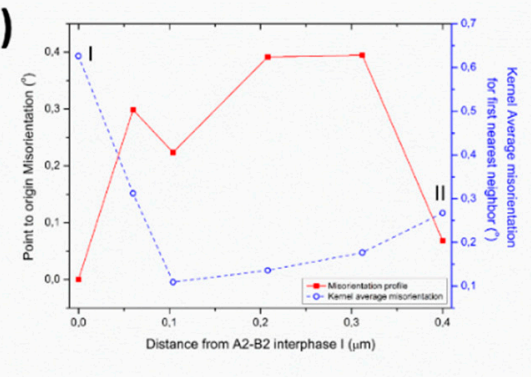

Figure 1. (a) Indent strain profile and (b) corresponding microstructure showing the dislocation motion across an A2/B2 interface. Dislocations experience strengthening when moving from the A2 to the ordered B2 phase, owing to spinodal and order hardening effects. The corresponding jerky dislocation motion is highlighted in nano-indentation load-displacement curves in (c). (d) shows the strain distribution with bright A2 phases being more plastic than the elastically stiffer dark B2 phase. (e) Corresponding misorientation profile and kernel average misorientation gradient on moving away from the interphase into the B2 phase. Figure 1d,e was adapted from [16] with permission from Elsevier, 2018. 


\subsection{Strengthening Mechanisms across Crystallographically Dissimilar Phase Boundaries}

Unlike grain boundaries, the mechanics of strengthening across heterophase interfaces involve diverse contributing factors. Classical grain boundaries govern strain transmission, primarily on the basis of grain boundary geometry and the alignment of active slip systems in pile-up and emission grains [28-31]. On the other hand, interphase boundaries involve interface-dependent strengthening mechanisms [32] that add to the overall extent of dislocation pile-up and internal stress configurations.

In theory, heterophase interphases derive strength from three main contributing mechanisms, apart from the geometric slip transmission criterion.

\subsubsection{Image forces or Koehler forces:}

The mismatch in shear moduli between neighboring phases gives rise to a Koehler force barrier between the dislocation and the interface $[33,34]$. The underlying effect that is responsible for this is the variation of strain energy per unit length of dislocation with changing modulus, such that a dislocation that moves from a stiffer grain to a softer grain would experience an attractive force, and the opposite scenario would result in repulsion between the incoming dislocation and the interface (Figure $2 \mathrm{c}$ ). Mathematically, Koehler forces $\left(\tau_{\text {Koehler }}\right)$ at an interphase boundary between phase $A$ and phase $B$ can be expressed as

$$
\tau_{\text {Koehler }}=\frac{G_{A}\left(G_{B}-G_{A}\right)}{4 \pi\left(G_{B}+G_{A}\right)} \cdot \frac{b}{h}
$$

where $G_{A}$ and $G_{B}$ are the shear moduli values of incident and emission grains, respectively; $b$ is the magnitude of the Burgers vector of active slip system in the incident grain; and $h$ is the normal distance between the dislocation and the interface. The exerted force is hypothetically similar to the stress field that is exerted by a negative image dislocation that is positioned at the other side of the interface, hence the term of image forces. The presence of Koehler forces significantly impacts the dislocation pile-up characteristics at the phase boundary that subsequently influence the strengthening that is imparted from the interfaces, as shown in Figure 2a,b. For instance, it has been shown in a BCC/FCC dual-phase $\mathrm{Al}_{\mathrm{x}} \mathrm{CoCrFeNi}$ alloy that the elastic modulus difference in FCC and ordered BCC phases $\left(\mathrm{E}_{\mathrm{BCC}}=275 \mathrm{GPa}\right.$ vs. $\left.\mathrm{E}_{\mathrm{FCC}}=252 \mathrm{GPa}\right)$ results in an attractive image force on incoming $\mathrm{BCC}$ dislocations and repulsive image forces on incoming FCC dislocations [35].

\subsubsection{Misfit Stresses}

Crystallographically dissimilar phase boundaries also result in interfacial stresses that arise from lattice parameter mismatch $(\Delta a)$ between adjacent phases (Figure $2 \mathrm{~d}$ ). The size misfit is compensated by a grid of van der Merwe dislocations that give rise to coherency strain hardening effects at the interface [36]. Coherency stresses typically dampen as a function of $1 / \lambda$ upon moving away from the interface, and they are mathematically given as,

$$
\tau_{\text {misfit }}=0.5 G^{*} \sqrt{\frac{2 b(\delta-\varepsilon)}{\lambda}}
$$

where $\delta=\frac{\Delta a}{\bar{a}} ; \bar{a}$ is the mean lattice parameter $\left(a_{\text {phase_} \_} A+a_{\text {phase_B } B}\right) / 2, \varepsilon=0.76 \delta$ is the residual elastic strain that was determined to agree for most heterophase interface types, $G^{*}$ is the average shear modulus for the two phases, and $\lambda$ is the grain dimension over which misfit stresses are determined, i.e., the distance between a dislocation and the interface. Coherency stresses exert a Peach-Koehler force on incoming glide dislocations, which can be either attractive or repulsive depending on the sense of applied stress with respect to the dislocation slip system. Apart from affecting the dislocation glide stresses, the coherency stresses aid in strengthening by additionally influencing the non-glide stress components of the dislocation stress field, whereby they can locally modify the dislocation core energy that directly influences the ease of a dislocation in overcoming an obstacle. 


\subsubsection{Chemical Mismatch Effect}

Another aspect that contributes to interfacial strengthening is a mismatch in chemical energy or gamma surfaces, as this mismatch directly determines the stacking fault energies in adjacent phases [37]. When the leading partial in a stacking fault moves across an interface, the dislocation configuration experiences an abrupt change in stacking fault energy. This manifests as an effective stress that is exerted upon the leading dislocation in the pile-up (Figure 2e). The resultant stacking fault strengthening stresses is described as

$$
\tau_{\text {chemical }}=\frac{\Delta \gamma}{b}
$$

where $\Delta \gamma$ is the stacking fault energy differential between neighboring phases. Overall, the change in energy of dislocation as it moves across the phase boundary involves an elastic energy contribution (that is a combination of $\tau_{\text {Koehler }}$ and $\tau_{\text {misfit }}$ ) and a chemical contribution in the form of $\tau_{\text {chemical }}$.

a)

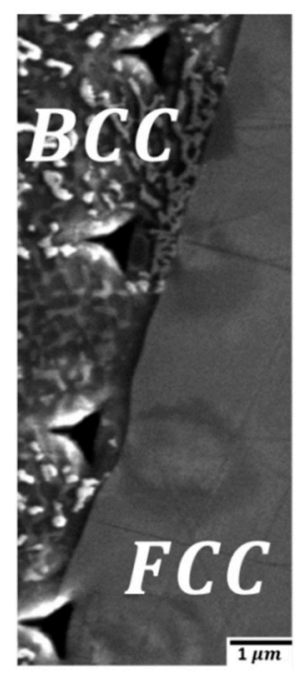

c)

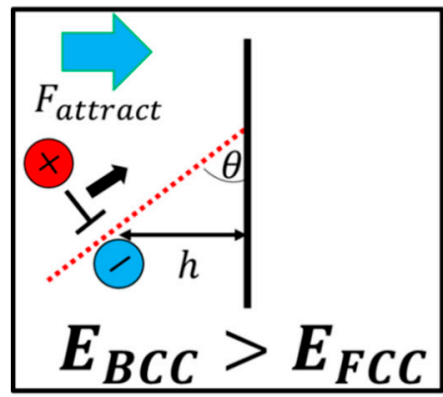

b)

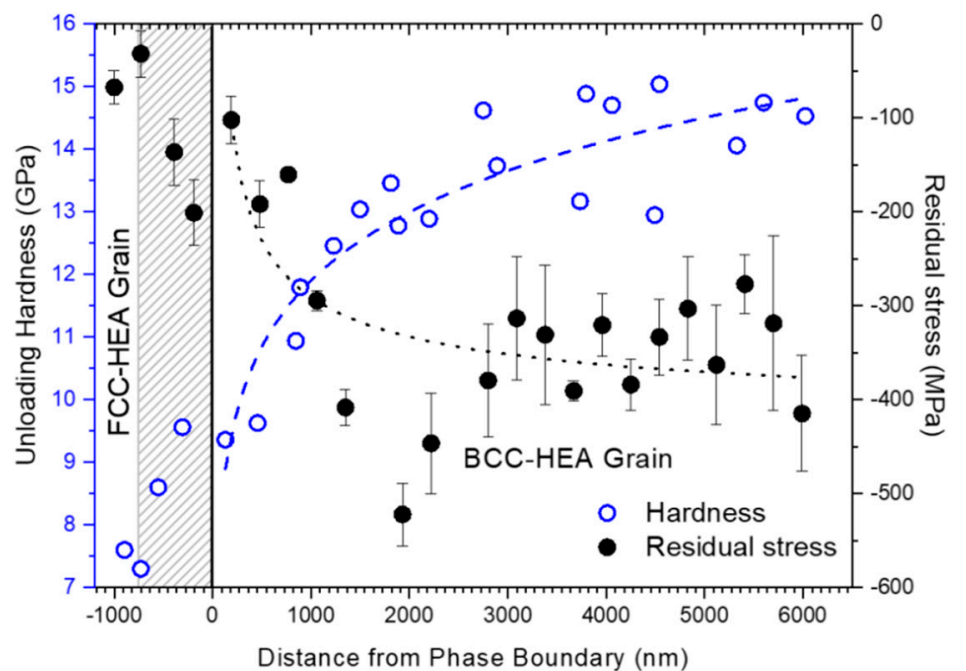

e)

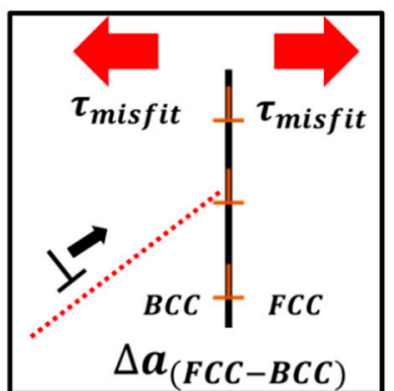

$\boldsymbol{\tau}_{\text {chemical }} \propto\left(\gamma_{S F(F C C)}-\gamma_{S F(B C C)}\right)$

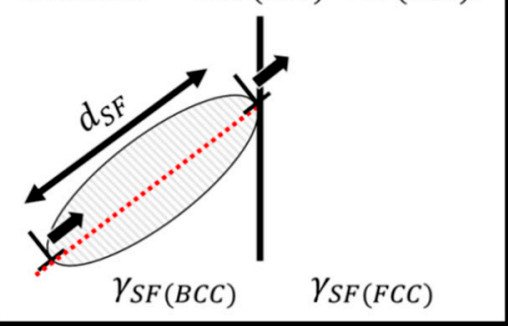

Figure 2. (a) BCC-FCC interface in an $\mathrm{Al}_{x} \mathrm{CoCrFeNi}$ high entropy alloy, with indent profile. (b) Variation of hardness and residual stress as a function of distance from phase boundary. The role of image forces is highlighted by local maximum in stress values on the BCC side (attractive image forces) and a local minimum on the FCC side (repulsive image forces). (c-e) illustrate different interfacial-dependent strengthening mechanisms. Figure $2 b$ was adapted from [2] with permission from Elsevier, 2018.

Owing to the strong compositional fluctuations and propensity of single-phase decomposition in HEAs, the contribution of interface-dependent strengthening could be significant in terms of augmenting overall material strength at both local and global scales. Recent studies have now 
made effective use of such hardening mechanisms to tailor microstructural designs that give rise to substantially stronger HEAs in comparison with their single-phase counterparts or with respect to conventional alloys. It has been shown that the BCC-FCC interfaces in HEAs could give rise to strengths of the order of $4 \mathrm{GPa}$ that are nearly four times the measured values of conventional BCC-FCC interfaces [35]. The underlying contribution for the augmented strengthening in HEAs has primarily been attributed to the enhanced interfacial-dependent strengthening that is caused by the strong compositional gradients in these alloys. Investigations on HEAs that comprised of BCC/FCC multilayers has indicated yield strengths of the order of 3.3. GPa, with more than two-thirds of this strength coming from interfacial strengthening effects and the remaining coming from solid solution strengthening [38]. In another study [39], it was shown that BCC/FCC interfacial strengthening mechanisms could be further enhanced by tuning the multilayer thickness in HEAs, whereby strengths of the order of $\sim 13 \mathrm{GPa}$ can be reached.

\section{An Outlook to HEAs: Structural Properties}

Compositionally, HEAs can be described as a concoction of multiple elements, an arrangement that often results in a frustrated crystal structure. Moreover, chemical gradients further trigger local rearrangements and the shuffling of elements, thus influencing the stability of the existing phases.

In short, HEAs are considerably more prone to phase transformation under applied temperature or stress, which could be a potent mechanism to trigger interesting plasticity mechanisms as well as to accommodate larger strains. For instance, in a seminal work by $\mathrm{Li}$ et al. on non-equiatomic compositions [10] based on the FCC single phase cantor alloy, it was shown that under plastic deformation, the dynamic transformation from an FCC to an HCP crystal structure is achieved that simultaneously enhances strength and ductility. In a more recent study [16], dynamic indentation-induced phase transition from $\mathrm{BCC}$ to $\mathrm{FCC}$ was observed in $\mathrm{BCC} \mathrm{Al}_{0.7} \mathrm{CoCrFeNi}$ HEAs (Figure $3 a, b$ ). The underlying reason behind the transformation was attributed to the spinodal decomposition of the BCC phase in Al, Ni rich-ordered B2 phases and random A2 phases (Figure 3a). Under applied stress, the A2 phases that are locally depleted in Al content could displacively transform and revert back to the more stable and ductile FCC phase (Figure 3c).

The results once again provide an opportunity to exploit the compositional fluctuations in tandem with thermomechanical treatment to dynamically trigger strength and ductility enhancing mechanisms. Displacive phase transformation effects or TRIP effects in HEAs could be exciting focal points in novel advances of HEAs in structural properties and applications.

Another mechanistic design criterion that employs compositional fluctuations is through the intrinsic modification of stacking fault energies. It was shown by Ritchie and co-workers [40,41] that by tuning local chemical ordering in HEAs, considerable variation in the intrinsic and extrinsic stacking fault energy values can be realized. Such design pathways are critical in terms of triggering new deformation mechanisms such as deformation twinning, whereby additional strain accommodation mechanisms are dynamically activated. Twinning not only contributes to plasticity but can also promote dynamic Hall-Petch-driven strengthening behavior, owing to grain fragmentation caused by twin boundary formation. For instance, it was shown by Deng et al. that for non-equiatomic $\mathrm{Fe}_{40} \mathrm{Mn}_{40} \mathrm{Co}_{10} \mathrm{Cr}_{10}$ HEA deformation, twinning is triggered as an additional mechanism for higher strains, thereby contributing to the overall strength-ductility increment [18].

Finally, the possibility to exploit simultaneous TRIP and TWIP effects along with multiscale strengthening effects that encompass interfacial strengthening and solid solution hardening effects can be envisioned in upcoming HEAs. In this regard, it has been shown that for the non-equiatomic FeMnCoCr alloy, dilute additions of C ( 0.6 at. \%) already trigger simultaneous twinning and phase transformation along with an interstitial hardening response [19]. Similar alloying strategies have been implemented on non-equiatomic BCC HfNbTaTiZr, wherein simultaneous plasticity-induced displacive transformation from the $\mathrm{BCC}$ phase to the HCP phase was observed, along with twinning in the HCP phase [42]. 
a)

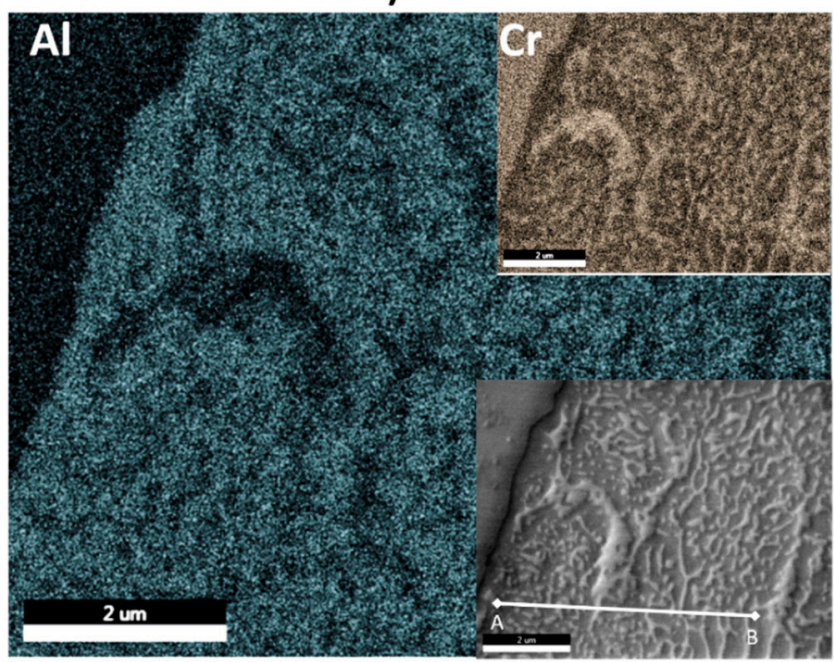

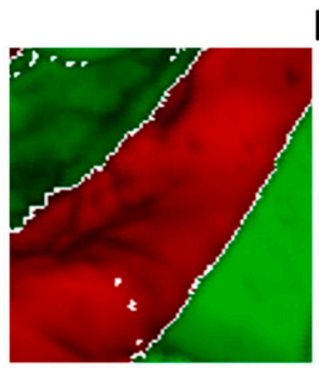

b)

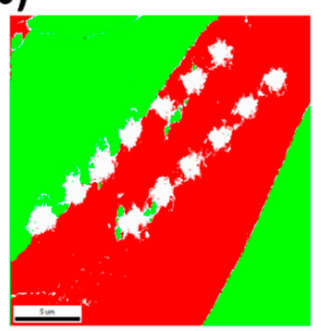

c)

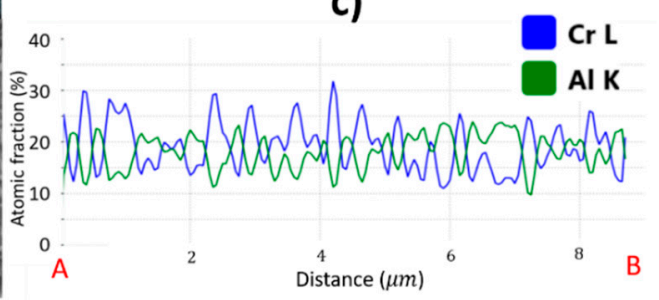

Figure 3. (a) EDS maps showing selective partitioning of $\mathrm{Al}$ and $\mathrm{Cr}$ into $\mathrm{B} 2$ and $\mathrm{A} 2$ phases, respectively. (b) Nano-indentation-induced phase transformation of A2 BCC phase (shown in red) to FCC phase (shown in green). Grain boundaries shown in white in the left image, and the non-indexed areas that are shown in white in the right image correspond to the experimentally made indents; local compositional fluctuations of $\mathrm{Al}$ and $\mathrm{Cr}$ indicated in (c), highlighting the instability of $\mathrm{A} 2 \mathrm{BCC}$ phases in regions of depleted Al, whereby such dynamic phase transformation to FCC at room temperature deformation is facilitated. Experimental data for the figures were derived from [16]. Figure 3b was adapted from [16] with permission from Elsevier, 2018.

The abovementioned impact of compositional fluctuations and strengthening modes related to phase formation also needs to be incorporated into current solid solution strengthening models in HEAs. There are now sufficient studies that have revealed that the strengthening of dislocation motion in HEAs is strongly dependent upon its susceptibility to display either short or long range ordering effects rather than simple lattice friction-induced hardening responses [43,44]. This was validated by a recent study by Robert Maaß and collaborators, wherein the peak dislocation velocities in $\mathrm{FCC} \mathrm{Al}_{0.3} \mathrm{CoCrFeNi}$ and pure $\mathrm{Au}$ did not show much difference, indicating that dislocation motion was not significantly sluggish in single phase solid solution HEAs (Rizzardi et al. [45]). Moreover, the contributions of interfacial-dependent strengthening and solute strengthening modes need to be appraised, as these could be critical in driving application-based future multiphase HEA alloy design. In this regard, greater efforts are needed in understanding the influence of alloying chemistry on engineering interphase boundaries in HEAs rather than focusing upon solid-solution strengthening as the primary strength contributor in these alloys. Indeed, the outcomes look promising and may open a new paradigm of structurally advanced HEAs, as was recently shown in study [46] where a compositionally graded $\mathrm{Al}_{\mathrm{x}} \mathrm{CoCrFeNi}$ bar was additively manufactured with increasing $\mathrm{Al}$ contents from $x=0.3$ to $x=0.7$ along the longitudinal direction, such that one end of the material was a single phase FCC and the other end formed a dual phase B2-FCC microstructure. From the point of view of mechanical response, the dual phase microstructure clearly highlighted the positive role of interfaces with significantly larger strengthening potentials compared to the single-phase FCC solid solutions.

\section{An Outlook to HEAs: Functional Properties}

Thus far, the emphasis in our feature paper has lied upon mechanical performance, plasticity and damage control. Attractive and rather unexplored frontiers of HEAs concern applications of functional properties, e.g., magnetic and electrical including thermoelectricity (Seebeck effect) [40], in the field of microelectronics and bio-medicine [23,47]. 
Several ideas are currently under investigation, and, in particular, we like to refer to possibilities of HEAs as cellular/porous materials that can be used in 'energy materials' (H-storage) but also explored as radiation resistant sensors and actuators. Actuation refers to mechanical displacement due to an electric signal. The opposite is also possible when an electrical current is generated by mechanical deformation, which creates what is known as piezoelectric materials. In general, these piezoelectrics need high voltages, with an order of $100 \mathrm{~V}$, and, at present, methods are being developed in medicine and biology to manufacture high-precision actuators that work at lower voltages on cell manipulation [48]. Structural stability over a large range of temperatures is essential.

In particular, highly porous metallic systems can mimic the properties of muscles upon an outside stimulus, and they have been coined 'artificial muscles' in analogy to human skeletal muscles, which are ideal actuators with a high energy efficiency, fast strain-rate response, and high durability. The common use of existing materials as actuators like piezoceramics and electroactive polymers are limited by several factors, including low energy efficiency, low strain amplitudes, fatigue limits, and the high actuation voltages needed. In our recent work, we have shown that nanoporous organometallic materials can operate as actuators, thereby offering a unique combination of relatively large strain amplitudes, high stiffness and strength, and importantly (see above) low operating voltages—say, at a few volts. However, a serious concern in this field of applications is (thermal) stability and the effects of coarsening.

Because of their high porosity and surface areas, the stability of cellular/porous systems is a major issue. In fact, depending on temperature (low versus high) and environment, the stability might be questionable, and, as a consequence, the functional properties might be not very stable and may deteriorate over time. Because of the suppression of the diffusional processes of defects at the surface of a (nano) ligament, we believe that HEAs might provide a very interesting and effective remedy to address these essential problems for applications of unique properties of functional HEA materials.

\subsection{Porous/Cellular Systems}

To illustrate the problem in a bit more detail, we discuss here the characteristics of cellular and porous media that possess a lower density and a higher surface area-to-volume ratio. The terminology is in macrofoams a bit different from that of nanofoams. In the former, besides pores, the material is made up of struts, and, in the latter, we call the struts ligaments. The topology of nodes and struts/ligaments can be anisotropic as well as isotropic. Besides these structural differences, nanoporous foams have been applied in nanofiltration systems, drug delivery platforms, catalysis, sensing and actuation [49-54]. In contrast, macro foams have been explored in macroscopic applications of the transport, automotive and aerospace industries.

A popular way of making metal nanofoams is based on dealloying through leaching. Preferably, the base material is a solid solution of a noble and a less noble element. Unfortunately, many alloys form intermetallics and many metals do not easily form solid solutions, which limits the dealloying methodology. Recently, nanoporous HEAs were produced through a rather novel method [55] by using liquid metal dealloying (LMD), a technique to fabricate non-noble porous materials by suppressing oxidation in a metallic melt [56-62]. It turned out that the structure of nanoporous TiVNbMoTa HEAs [55] can be described as nanoscale ligaments of a solid-solution phase, the stability of which is due to suppressed surface diffusion.

\subsection{Suppression of Coarsening}

It should be realized that the actuation mechanism based on nanoporous metals with high surface-to-volume ratios is different to piezoceramics. For details, one can look to [63]. The physical principles in the case of metals are based on the lower coordination of the surface atoms. Therefore, to gain 'density,' the atoms move inwards, and a positive displacement is necessary to bring them back to the equilibrium interatomic distances of the situation in the bulk. Therefore, a positive, i.e., tensile stress state at the surface, is generated that is compensated for by a compressive stress state inside the 
strut/ligament. Clearly, a positive charge injection can equilibrate the existing excess negative surface charge. As a consequence, a positive charge lowers the positive tensile stress and, importantly, relaxes the negative compressive stress in the ligament, i.e., the negative compressive stress becomes a bit more 'positive', i.e., a positive charge will generate a positive displacement that can be detected by optical means by using a small laser. It is noteworthy that these rather small displacements as a result of stress relaxations are not detectable in macrofoams, but nanofoams are completely different because a much large surface area to volume ratio exists. To give a rough estimate: If the size of the ligaments is of the order of $5-10 \mathrm{~nm}$, a substantial fraction of the total number of atoms, say $20 \%-10 \%$, is on the surface, meaning that a $100 \mathrm{~nm}$ thick ligament already reflects the bulk situation, and the actuating properties are hardly detectable. It is important to realize that the electronic charge distribution at a nanoporous metal interface can effectively be controlled during cycling voltammetry experiments. Only small electrical voltages of the order of $1 \mathrm{~V}$ are needed to bring positive or negative charge carriers (ions) from the electrolyte to the nanoporous metal [64,65]. Of course, working with liquid electrolytes can be cumbersome, and [65] we recently demonstrated that metallic muscles can operate in a dry environment, even at high strain rates, i.e., much higher than allowed for in electrochemical artificial muscles [65].

Nonetheless, electrochemical processes may lead to severe coarsening (undesired growth) of the ligaments [66-68], which is a major concern because actuation is hampered or completely lost, as is shown in Figure 4, where strain amplitudes are plotted as a function of the average ligament size. As expected, there has been a strong size effect and the strain amplitude recorded on nanoporous systems with different ligament sizes, and these may decrease upon increasing the ligament size (Figure 4). Even as a sensor (i.e., without external applied electric fields), the response is not stable due to coarsening effects (Figure 5), and, at higher temperatures, these effects are amplified tremendously.

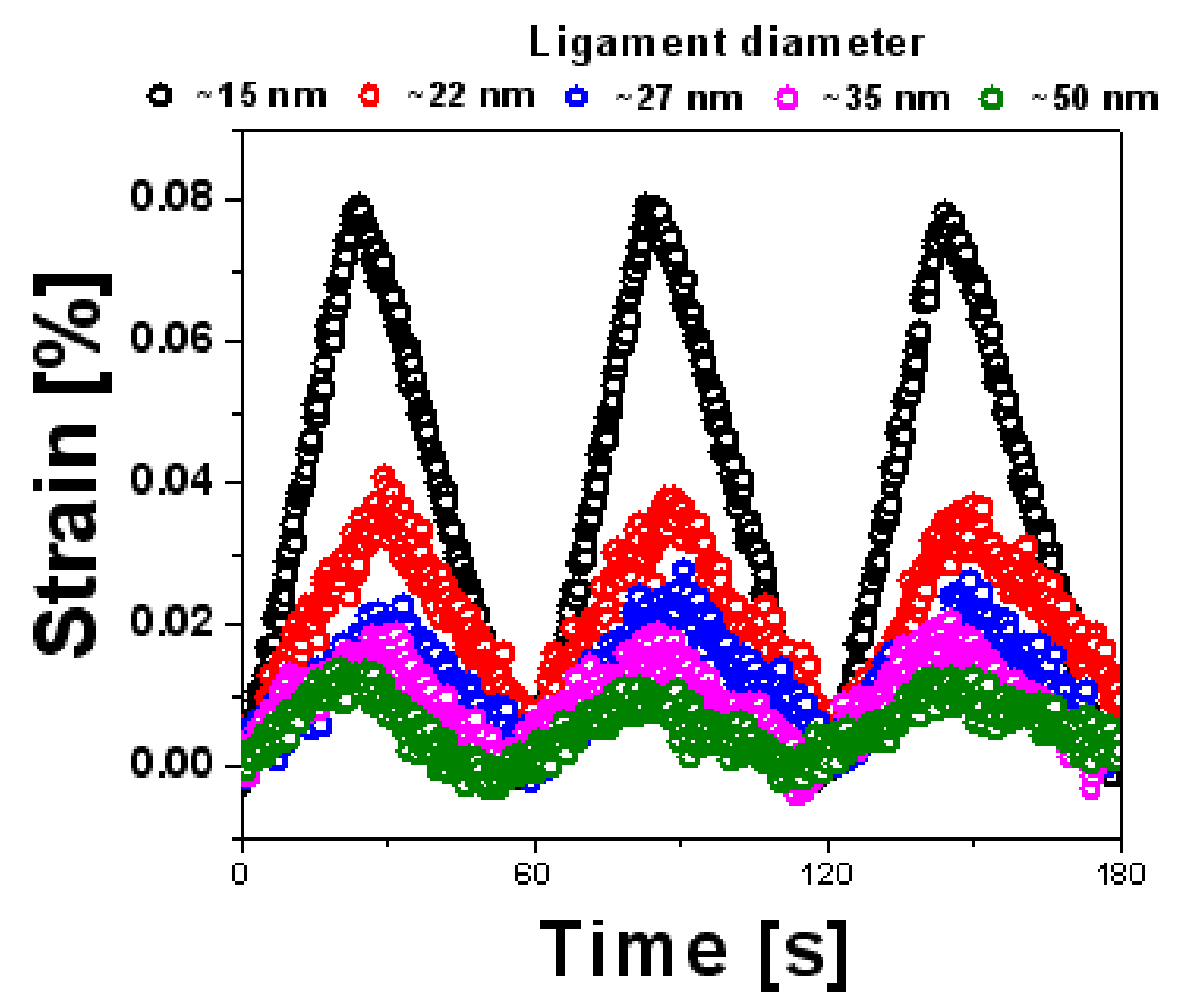

Figure 4. Ligament size-dependence of the charge-induced strain in nanoporous metals (Au). The strain amplitude that was recorded on five specimens with different ligament sizes decreased with increasing ligament size. This shows that ligament growth during electrochemical actuation is undesirable $[67,68]$. 

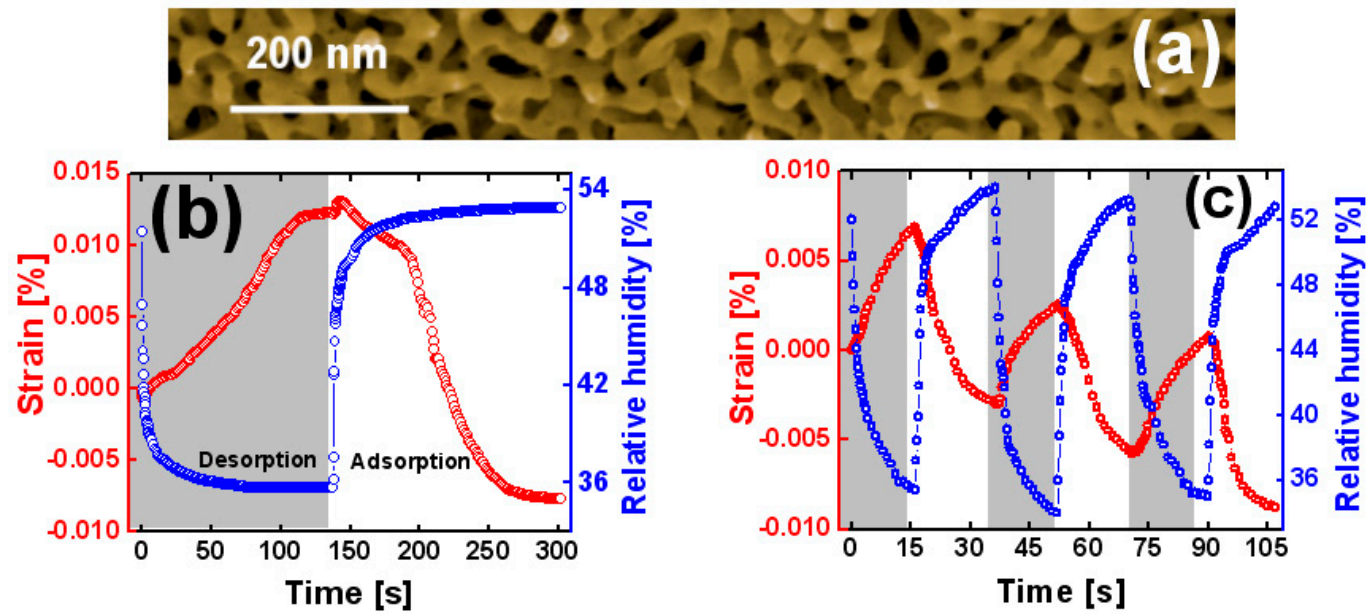

Figure 5. (a) Typical scanning electron micrograph of a nanoporous material (Au) that was synthesized by the dealloying process. The diameter of the ligaments was about $20 \mathrm{~nm}$. (b) Display of the changes in relative humidity versus time for alternations of humid and dry air (blue curve refers to right ordinate) and corresponding strain versus time (red curve refers to left ordinate). (c) Responses of relative humidity versus time for long alternations of dry and humid air (blue curve refers to right ordinate) and corresponding strain versus time (red curve refers to left ordinate [67]).

The solution to these instabilities is basically to reduce surface diffusion, and, for that reason, HEAs may offer a suitable solution. Recently, Soo-Hyun Joo and collaborators found an exceptional stability against coarsening of an MoNbTaTiV nanoporous HEA at elevated temperatures [55]. The ligament size and distribution of the HEA versus dealloying time at various temperatures are displayed in Figure 6.

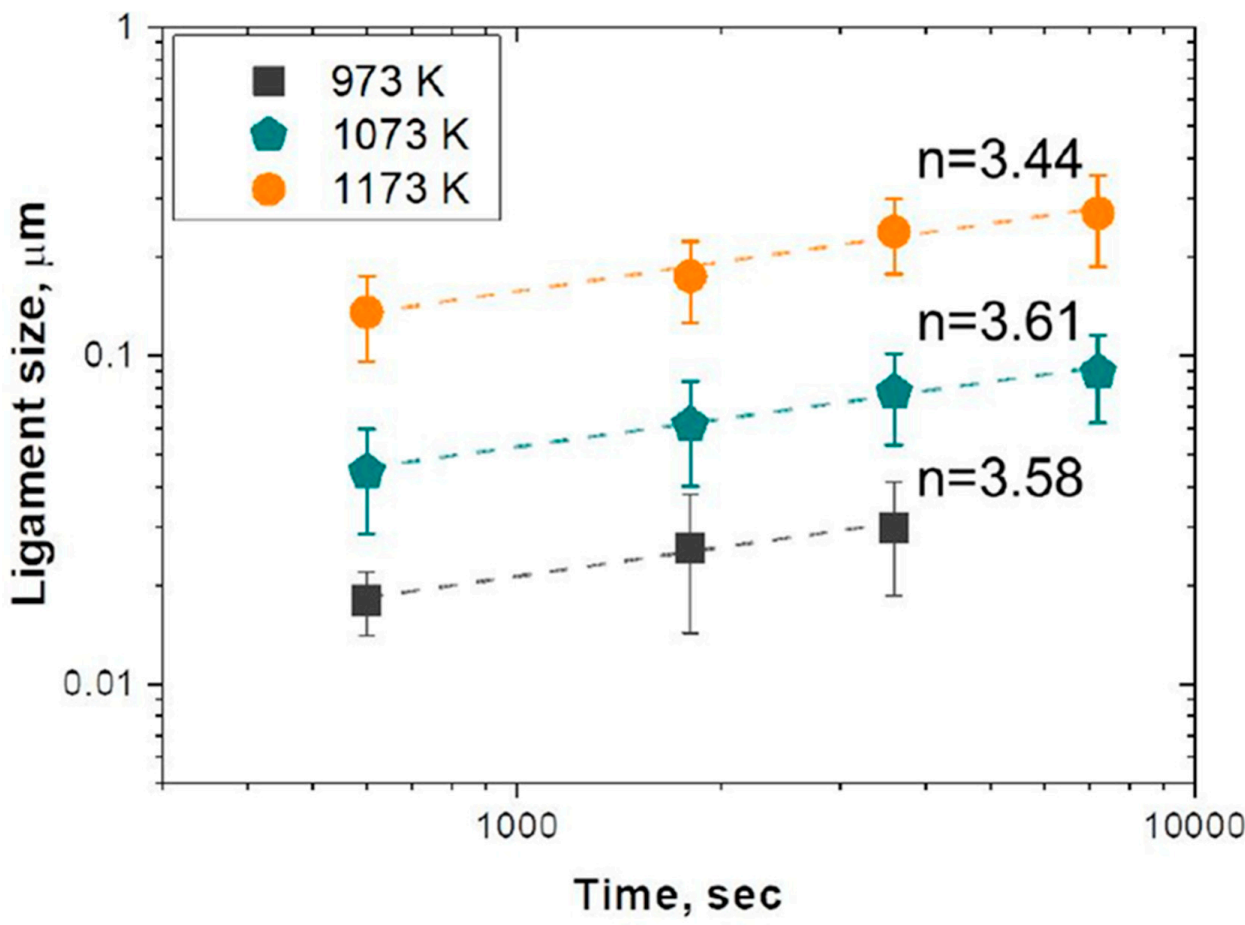

Figure 6. Ligament size in a TiVNbMoTa nanoporous high entropy alloy (HEA) versus dealloying time at various temperatures. The dealloying time, $t$, and ligament size, $d$, are correlated through a power function $d^{n}=k t D$, where $n$ is the coarsening component, $k$ is a constant, and $D$ is the surface diffusivity. By plotting the $\ln [d(t)]$ vs. $\ln t$ curve, the coarsening exponent, $n$, can be obtained. Error bars denote the distribution of ligament sizes. (Figure reprinted from [55] with permission from Wiley, 2019.) 
To make the coarsening behavior a bit more quantitative: Dealloying time, $t$, and ligament size, $d$, are written as a power function: $d^{n}=k t D$, where $n$ is the coarsening component, $k$ is a constant, and $D$ is the surface diffusivity. By plotting the $\ln [d(t)]$ vs. $\ln t$ curve, the coarsening exponent, $n$, can be measured. Figure 6 displays the measured specific surface areas of $55.7(<10 \mathrm{~nm}), 38.8(14 \mathrm{~nm})$, and 3.6 $(155 \mathrm{~nm}) \mathrm{m}^{2} / \mathrm{g}$, depending on the ligament size as obtained in [55] based on an analytical model we designed in the past [68]. The adsorption/desorption isotherm curve (inset in Figure 7) corresponds to a ligament size of about $10 \mathrm{~nm}$. The hysteresis loop in the isotherm is associated with capillary condensation in nanopores $(<50 \mathrm{~nm})$.

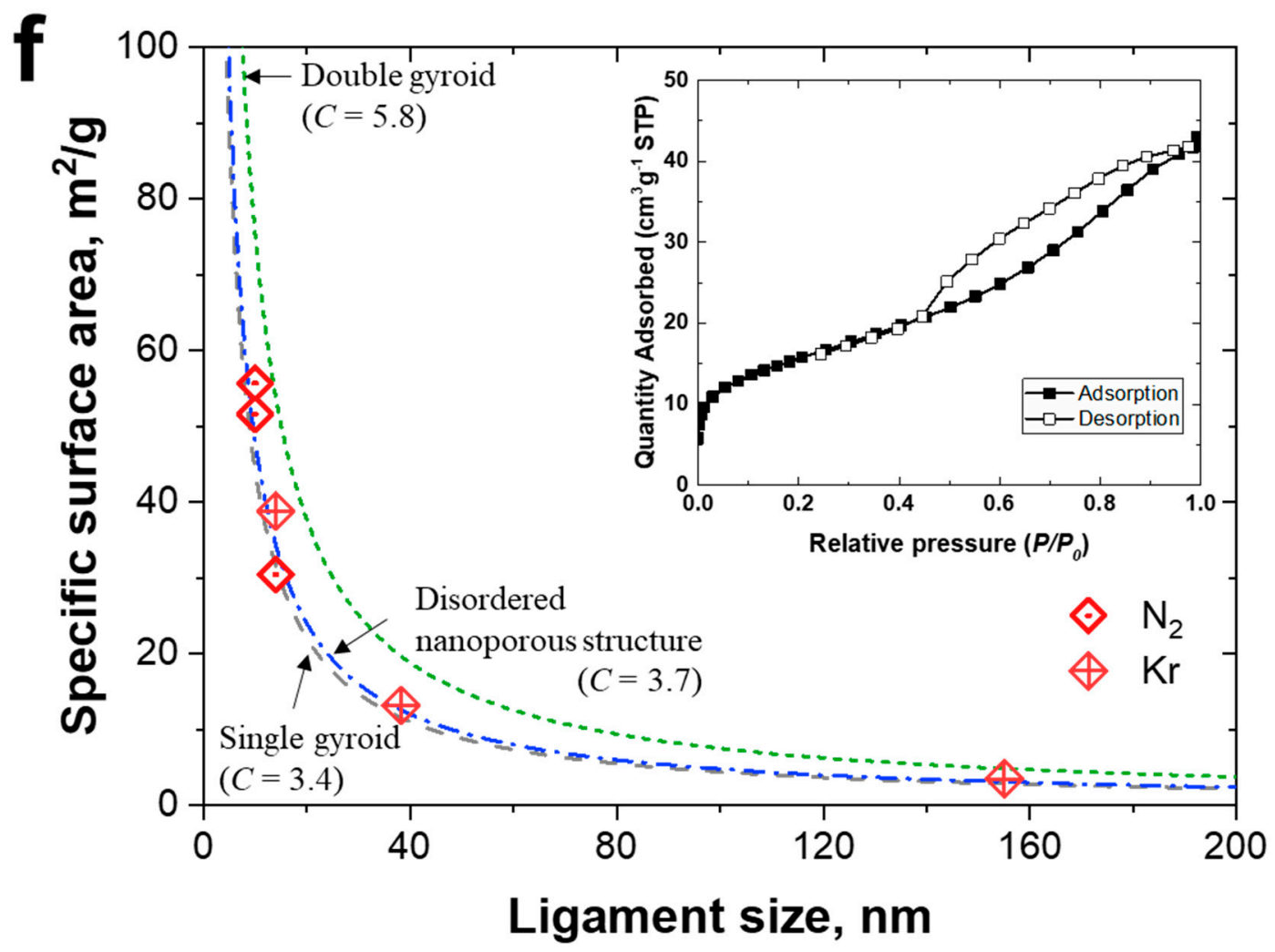

Figure 7. Specific surface areas measured with the Brunauer-Emmett-Teller (BET) method as a function of the ligament size in a TiVNbMoTa nanoporous HEA. The lines (dashed and dashed-dot) correspond to the predictions based on the analytic model $S=C / \rho d$, as designed and tested in [68]. The inset shows the nitrogen adsorption/desorption curves with an average ligament size of $\sim 10 \mathrm{~nm}$. The solid bulk density $\rho$ was assumed to be $7.7 \mathrm{~g} \cdot \mathrm{cm}^{-3}$ from the average atomic weight and the radius of the constituent elements (Figure reprinted from [55] with permission from Wiley, 2019.)

As far as the physical explanations of these phenomena are concerned, a first and easy conclusion would be: The suppression of coarsening is due to what has been called sluggish diffusion in HEA. However, this might be a too simple and naïve reason. There is no literature on the details of diffusional processes in nano-porous HEA, but many papers have been published on bulk HEA [69], e.g., CoCrFeMnNi [70]. Dezso Beke [71] analyzed the diffusion coefficients of elements in CoCrFeMnNi HEA. Interestingly, sluggish diffusion could be explained as not being based on high activation energies but on correlation effects. Indeed [72], it has been reported that the diffusion of Ni in both HEAs follow an Arrhenius behavior. In fact, the tracer diffusion in HEAs does not become sluggish at absolute temperature; it only becomes so if it is considered at a homologous temperature. As a sequence, it can be concluded that diffusion in HEAs is not sluggish, as such, but other factors such as frequency factors can explain a slower diffusion rate in HEAs. From a scientific viewpoint, more in-depth analyses regarding the diffusional processes are necessary, not only for bulk HEA but also for surface diffusional processes in nanoporous HEAs. 
A stimulating and exciting field of research would be to devise specific rules for the design of highly porous HEAs that can be used over a large range of temperatures in the field of sensors and actuators, in 'energy materials' (H-storage), radiation resistant materials, and many other scenarios. It is important to note that in these cellular and highly porous HEAs materials, it is not just the specific materials properties of strength and surface diffusion matter-the local topology and connectivity of struts and nano-ligaments also does [73].

As a consequence, topology provides an additional design parameter, in addition to the extrinsic and intrinsic material size-dependent properties [73,74]. This approach fits the term 'architected material,' which was, to the best of our knowledge, proposed for the first time by Mike Ashby and Yves Bréchet and which bridges the structural engineering of topology and good practice in architecture [75]. More recently, a special issue of excellent contributions to the Materials Research Society (MRS) Bulletin, edited by Julia R. Greer and Vikram S. Deshpande [76], was published on the design, fabrication and mechanical performance of three-dimensional architected materials and structures. It would be exciting to explore HEAs among these lines of 'architected materials' for the optimization of the 'integral' of both structural and functional properties together for novel applications.

Funding: This research received no external funding.

Acknowledgments: The work was supported by the Applied Physics-Materials Science group of the Zernike Institute for Advanced Materials of the University of Groningen, the Netherlands. Discussions and contributions by Eric Detsi (now at UPENN, Philadelphia, USA) and Václav Ocelík (Groningen, the Netherlands) are gratefully acknowledged.

Conflicts of Interest: The authors declare no conflict of interest.

\section{References}

1. Tsai, M.-H.; Yeh, J.-W. High-Entropy Alloys: A Critical Review. Mater. Res. Lett. 2014, 2, 107-123. [CrossRef]

2. Yeh, J.-W.; Chen, S.-K.; Lin, S.-J.; Gan, J.-Y.; Chin, T.-S.; Shun, T.-T.; Tsau, C.-H.; Chang, S.-Y. Nanostructured High-Entropy Alloys with Multiple Principal Elements: Novel Alloy Design Concepts and Outcomes. Adv. Eng. Mater. 2004, 6, 299-303. [CrossRef]

3. Ranganathan, S. Alloyed pleasures: Multimetallic cocktails. Curr. Sci. 2003, 85, 1404-1406.

4. Christofidou, K.A.; Pickering, E.J.; Orsatti, P.; Mignanelli, P.M.; Slater, T.J.A.; Stone, H.J.; Jones, N.G. On the influence of $\mathrm{Mn}$ on the phase stability of the CrMnxFeCoNi high entropy alloys. Intermetallics 2018, 92, 84-92. [CrossRef]

5. Pickering, E.J.; Jones, N.G. High-entropy alloys: A critical assessment of their founding principles and future prospects. Int. Mater. Rev. 2016, 61, 183-202. [CrossRef]

6. Pickering, E.J.; Muñoz-Moreno, R.; Stone, H.J.; Jones, N.G. Precipitation in the equiatomic high-entropy alloy CrMnFeCoNi. Scr. Mater. 2016, 113, 106-109. [CrossRef]

7. Jones, N.G.; Frezza, A.; Stone, H.J. Phase equilibria of an A10.5CrFeCoNiCu High Entropy Alloy. Mater. Sci. Eng. A 2014, 615, 214-221. [CrossRef]

8. Jones, N.G.; Izzo, R.; Mignanelli, P.M.; Christofidou, K.A.; Stone, H.J. Phase evolution in an A10.5CrFeCoNiCu High Entropy Alloy. Intermetallics 2016, 71, 43-50. [CrossRef]

9. Senkov, O.N.; Miller, J.D.; Miracle, D.B.; Woodward, C. Accelerated exploration of multi-principal element alloys with solid solution phases. Nat. Commun. 2015, 6, 6529. [CrossRef]

10. Li, Z.; Pradeep, K.G.; Deng, Y.; Raabe, D.; Tasan, C.C. Metastable high-entropy dual-phase alloys overcome the strength-ductility trade-off. Nature 2016, 534, 227-230. [CrossRef]

11. Otto, F.; Dlouhý, A.; Pradeep, K.G.; Kuběnová, M.; Raabe, D.; Eggeler, G.; George, E.P. Decomposition of the single-phase high-entropy alloy $\mathrm{CrMnFeCoNi}$ after prolonged anneals at intermediate temperatures. Acta Mater. 2016, 112, 40-52. [CrossRef]

12. Otto, F.; Yang, Y.; Bei, H.; George, E.P. Relative effects of enthalpy and entropy on the phase stability of equiatomic high-entropy alloys. Acta Mater. 2013, 61, 2628-2638. [CrossRef]

13. George, E.P.; Raabe, D.; Ritchie, R.O. High-entropy alloys. Nat. Rev. Mater. 2019, 4, 515-534. [CrossRef]

14. Basu, I.; Ocelík, V.; De Hosson, J.T.M. Size effects on plasticity in high-entropy alloys. J. Mater. Res. 2018, 33, 3055-3076. [CrossRef] 
15. Miracle, D.B. High entropy alloys as a bold step forward in alloy development. Nat. Commun. 2019, 10, 1805. [CrossRef] [PubMed]

16. Basu, I.; Ocelík, V.; De Hosson, J.T.M. Size dependent plasticity and damage response in multiphase body centered cubic high entropy alloys. Acta Mater. 2018, 150, 104-116. [CrossRef]

17. He, J.Y.; Wang, H.; Huang, H.L.; Xu, X.D.; Chen, M.W.; Wu, Y.; Liu, X.J.; Nieh, T.G.; An, K.; Lu, Z.P. A precipitation-hardened high-entropy alloy with outstanding tensile properties. Acta Mater. 2016, 102, 187-196. [CrossRef]

18. Deng, Y.; Tasan, C.C.; Pradeep, K.G.; Springer, H.; Kostka, A.; Raabe, D. Design of a twinning-induced plasticity high entropy alloy. Acta Mater. 2015, 94, 124-133. [CrossRef]

19. Li, Z.; Tasan, C.C.; Springer, H.; Gault, B.; Raabe, D. Interstitial atoms enable joint twinning and transformation induced plasticity in strong and ductile high-entropy alloys. Sci. Rep. 2017, 7, 40704. [CrossRef]

20. Gludovatz, B.; Hohenwarter, A.; Catoor, D.; Chang, E.H.; George, E.P.; Ritchie, R.O. A fracture-resistant high-entropy alloy for cryogenic applications. Science 2014, 345, 1153-1158. [CrossRef]

21. Li, Z.; Zhao, S.; Ritchie, R.O.; Meyers, M.A. Mechanical properties of high-entropy alloys with emphasis on face-centered cubic alloys. Prog. Mater. Sci. 2019, 102, 296-345. [CrossRef]

22. Zhang, Z.; Mao, M.M.; Wang, J.; Gludovatz, B.; Zhang, Z.; Mao, S.X.; George, E.P.; Yu, Q.; Ritchie, R.O. Nanoscale origins of the damage tolerance of the high-entropy alloy CrMnFeCoNi. Nat. Commun. 2015, 6, 10143. [CrossRef] [PubMed]

23. Zhang, Y.; Zuo, T.T.; Tang, Z.; Gao, M.C.; Dahmen, K.A.; Liaw, P.K.; Lu, Z.P. Microstructures and properties of high-entropy alloys. Prog. Mater. Sci. 2014, 61,1-93. [CrossRef]

24. Rao, J.C.; Ocelík, V.; Vainchtein, D.; Tang, Z.; Liaw, P.K.; De Hosson, J.T.M. The fcc-bcc crystallographic orientation relationship in AlxCoCrFeNi high-entropy alloys. Mater. Lett. 2016, 176, 29-32. [CrossRef]

25. Wang, W.-R.; Wang, W.-L.; Wang, S.-C.; Tsai, Y.-C.; Lai, C.-H.; Yeh, J.-W. Effects of Al addition on the microstructure and mechanical property of AlxCoCrFeNi high-entropy alloys. Intermetallics 2012, 26, 44-51. [CrossRef]

26. Soni, V.; Senkov, O.N.; Gwalani, B.; Miracle, D.B.; Banerjee, R. Microstructural Design for Improving Ductility of An Initially Brittle Refractory High Entropy Alloy. Sci. Rep. 2018, 8, 8816. [CrossRef]

27. Senkov, O.N.; Isheim, D.; Seidman, D.N.; Pilchak, A.L. Development of a Refractory High Entropy Superalloy. Entropy 2016, 18, 102. [CrossRef]

28. Clark, W.A.T.; Wagoner, R.H.; Shen, Z.Y.; Lee, T.C.; Robertson, I.M.; Birnbaum, H.K. On the criteria for slip transmission across interfaces in polycrystals. Scr. Metall. Mater. 1992, 26, 203-206. [CrossRef]

29. Shen, Z.; Wagoner, R.H.; Clark, W.A.T. Dislocation and grain boundary interactions in metals. Acta Metall. 1988, 36, 3231-3242. [CrossRef]

30. Kacher, J.; Eftink, B.P.; Cui, B.; Robertson, I.M. Dislocation interactions with grain boundaries. Curr. Opin. Solid State Mater. Sci. 2014, 18, 227-243. [CrossRef]

31. Basu, I.; Ocelík, V.; De Hosson, J.T.M. Measurement of spatial stress gradients near grain boundaries. Scr. Mater. 2017, 136, 11-14. [CrossRef]

32. Rao, S.I.; Hazzledine, P.M. Atomistic simulations of dislocation-interface interactions in the Cu-Ni multilayer system. Philos. Mag. A 2000, 80, 2011-2040. [CrossRef]

33. Head, A.K.X. The Interaction of Dislocations and Boundaries. London Edinburgh Dublin Philos. Mag. J. Sci. 1953, 44, 92-94. [CrossRef]

34. Koehler, J.S. Attempt to Design a Strong Solid. Phys. Rev. B 1970, 2, 547-551. [CrossRef]

35. Basu, I.; Ocelík, V.; De Hosson, J.T. BCC-FCC interfacial effects on plasticity and strengthening mechanisms in high entropy alloys. Acta Mater. 2018, 157, 83-95. [CrossRef]

36. Frank, F.C.; Van der Merwe, J. One-dimensional dislocations. II. Misfitting monolayers and oriented overgrowth. Proc. R. Soc. London Ser. A Math. Phys. Sci. 1949, 198, 216-225.

37. Vitek, V. Theory of the core structures of dislocations in BCC metals. Cryst. Lattice Defects 1974, 5, 1-34.

38. Cai, Y.P.; Wang, G.J.; Ma, Y.J.; Cao, Z.H.; Meng, X.K. High hardness dual-phase high entropy alloy thin films produced by interface alloying. Scripta Mater. 2019, 162, 281-285. [CrossRef]

39. Cao, Z.H.; Ma, Y.J.; Cai, Y.P.; Wang, G.J.; Meng, X.K. High strength dual-phase high entropy alloys with a tunable nanolayer thickness. Scripta Materialia 2019, 173, 149-153. [CrossRef]

40. Ding, J.; Yu, Q.; Asta, M.; Ritchie, R.O. Tunable stacking fault energies by tailoring local chemical order in CrCoNi medium-entropy alloys. Proc. Natl. Acad. Sci. USA 2018, 115, 8919-8924. [CrossRef] 
41. Ding, Q.; Zhang, Y.; Chen, X.; Fu, X.; Chen, D.; Chen, S.; Gu, L.; Wei, F.; Bei, H.; Gao, Y.; et al. Tuning element distribution, structure and properties by composition in high-entropy alloys. Nature 2019, 574, 223-227. [CrossRef] [PubMed]

42. Lilensten, L.; Couzinié, J.-P.; Bourgon, J.; Perrière, L.; Dirras, G.; Prima, F.; Guillot, I. Design and tensile properties of a bcc Ti-rich high-entropy alloy with transformation-induced plasticity. Mater. Res. Lett. 2017, 5, 110-116. [CrossRef]

43. Bracq, G.; Laurent-Brocq, M.; Varvenne, C.; Perrière, L.; Curtin, W.A.; Joubert, J.M.; Guillot, I. Combining experiments and modeling to explore the solid solution strengthening of high and medium entropy alloys. Acta Mater. 2019, 177, 266-279. [CrossRef]

44. Osetsky, Y.N.; Pharr, G.M.; Morris, J.R. Two modes of screw dislocation glide in fcc single-phase concentrated alloys. Acta Mater. 2019, 164, 741-748. [CrossRef]

45. Rizzardi, Q.; Sparks, G.; Maaß, R. Fast Slip Velocity in a High-Entropy Alloy. JOM 2018, 70, $1088-1093$. [CrossRef]

46. Gwalani, B.; Gangireddy, S.; Shukla, S.; Yannetta, C.J.; Valentin, S.G.; Mishra, R.S.; Banerjee, R. Compositionally graded high entropy alloy with a strong front and ductile back. Mater. Today Commun. 2019, 20, 100602.

47. Miracle, D.B. Critical Assessment 14: High entropy alloys and their development as structural materials. Mater. Sci. Technol. 2015, 31, 1142-1147. [CrossRef]

48. Kudoh, K. Development of piezo micromanipulator for cell micromanipulation. J. Mamm. Ova Res. 1990, 7, 7-12.

49. Wittstock, A.; Zielasek, V.; Biener, J.; Friend, C.M.; Bäumer, M. Nanoporous Gold Catalysts for Selective Gas-Phase Oxidative Coupling of Methanol at Low Temperature. Science 2010, 327, 319. [CrossRef]

50. Nagle, L.C.; Rohan, J.F. Nanoporous gold anode catalyst for direct borohydride fuel cell. Int. J. Hydrogen Energy 2011, 36, 10319-10326. [CrossRef]

51. Lang, X.Y.; Yuan, H.T.; Iwasa, Y.; Chen, M.W. Three-dimensional nanoporous gold for electrochemical supercapacitors. Scripta Mater. 2011, 64, 923-926. [CrossRef]

52. Detsi, E.; Chen, Z.G.; Vellinga, W.P.; Onck, P.R.; Hosson, J.T.M.D. Actuating and Sensing Properties of Nanoporous Gold. J. Nanosci. Nanotechnol. 2012, 12, 4951-4955. [CrossRef] [PubMed]

53. Yavuz, M.S.; Cheng, Y.; Chen, J.; Cobley, C.M.; Zhang, Q.; Rycenga, M.; Xie, J.; Kim, C.; Song, K.H.; Schwartz, A.G.; et al. Gold nanocages covered by smart polymers for controlled release with near-infrared light. Nat. Mater. 2019, 8, 935-939. [CrossRef]

54. Au, L.; Zheng, D.; Zhou, F.; Li, Z.-Y.; Li, X.; Xia, Y. A Quantitative Study on the Photothermal Effect of Immuno Gold Nanocages Targeted to Breast Cancer Cells. ACS Nano 2008, 2, 1645-1652. [CrossRef] [PubMed]

55. Joo, S.-H.; Bae, J.W.; Park, W.-Y.; Shimada, Y.; Wada, T.; Kim, H.S.; Takeuchi, A.; Konno, T.J.; Kato, H.; Okulov, I.V. Beating Thermal Coarsening in Nanoporous Materials via High-Entropy Design. Adv. Mater. 2019, 1906160. [CrossRef]

56. Wada, T.; Yubuta, K.; Inoue, A.; Kato, H. Dealloying by metallic melt. Mater. Lett. 2011, 65, 1076-1078. [CrossRef]

57. Wada, T.; Ichitsubo, T.; Yubuta, K.; Segawa, H.; Yoshida, H.; Kato, H. Bulk-Nanoporous-Silicon Negative Electrode with Extremely High Cyclability for Lithium-Ion Batteries Prepared Using a Top-Down Process. Nano Lett. 2014, 14, 4505-4510. [CrossRef]

58. Geslin, P.-A.; McCue, I.; Gaskey, B.; Erlebacher, J.; Karma, A. Topology-generating interfacial pattern formation during liquid metal dealloying. Nat. Commun. 2015, 6, 8887. [CrossRef]

59. Okulov, I.V.; Weissmüller, J.; Markmann, J. Dealloying-based interpenetrating-phase nanocomposites matching the elastic behavior of human bone. Sci. Rep. 2017, 7, 20. [CrossRef]

60. Okulov, I.V.; Okulov, A.V.; Soldatov, I.V.; Luthringer, B.; Willumeit-Römer, R.; Wada, T.; Kato, H.; Weissmüller, J.; Markmann, J. Open porous dealloying-based biomaterials as a novel biomaterial platform. Mater. Sci. Eng. C 2018, 88, 95-103. [CrossRef]

61. Joo, S.-H.; Wada, T.; Kato, H. Development of porous FeCo by liquid metal dealloying: Evolution of porous morphology and effect of interaction between ligaments and melt. Mater. Des. 2019, 180, 107908.

62. Joo, S.-H.; Yubuta, K.; Kato, H. Ordering kinetics of nanoporous FeCo during liquid metal dealloying and the development of nanofacets. Scr. Mater. 2020, 177, 38-43. [CrossRef]

63. Jayachandran, K.P.; Guedes, J.M.; Rodrigues, H.C. Ferroelectric materials for piezoelectric actuators by optimal design. Acta Mater. 2011, 59, 3770-3778. [CrossRef] 
64. Saane, S.S.R.; Mangipudi, K.R.; Loos, K.U.; De Hosson, J.T.M.; Onck, P.R. Multiscale modeling of charge-induced deformation of nanoporous gold structures. J. Mech. Phys. Solids 2014, 66, 1-15. [CrossRef]

65. Detsi, E.; Punzhin, S.; Rao, J.; Onck, P.R.; De Hosson, J.T.M. Enhanced Strain in Functional Nanoporous Gold with a Dual Microscopic Length Scale Structure. ACS Nano 2012, 6, 3734-3744. [CrossRef] [PubMed]

66. Zhang, J.; Liu, P.; Ma, H.; Ding, Y. Nanostructured Porous Gold for Methanol Electro-Oxidation. J. Phys. Chem. C 2007, 111, 10382-10388. [CrossRef]

67. Detsi, E.; Onck, P.; De Hosson, J.T.M. Metallic Muscles at Work: High Rate Actuation in Nanoporous Gold/Polyaniline Composites. ACS Nano 2013, 7, 4299-4306. [CrossRef]

68. Detsi, E.; De Jong, E.; Zinchenko, A.; Vuković, Z.; Vuković, I.; Punzhin, S.; Loos, K.; ten Brinke, G.; De Raedt, H.A.; Onck, P.R.; et al. On the specific surface area of nanoporous materials. Acta Mater. 2011, 59, 7488-7497. [CrossRef]

69. Praveen, S.; Kim, H.S. High-Entropy Alloys: Potential Candidates for High-Temperature Applications-An Overview. Adv. Eng. Mater. 2018, 20, 1700645. [CrossRef]

70. Tsai, K.Y.; Tsai, M.H.; Yeh, J.W. Sluggish diffusion in Co-Cr-Fe-Mn-Ni high-entropy alloys. Acta Mater. 2013, $61,4887-4897$.

71. Beke, D.L.; Erdélyi, G. On the diffusion in high-entropy alloys. Mater. Lett. 2016, 164, 111-113. [CrossRef]

72. Vaidya, M.; Trubel, S.; Murty, B.S.; Wilde, G.; Divinski, S.V. Ni tracer diffusion in CoCrFeNi and CoCrFeMnNi high entropy alloys. J. Alloys Compd. 2016, 688, 994-1001. [CrossRef]

73. Michelsen, K.; De Raedt, H.; De Hosson, J.T.M. Aspects of mathematical morphology. In Advances in Imaging and Electron Physics; Hawkes, P.W., Kazan, B., Mulvey, T., Eds.; Elsevier: Amsterdam, Netherlands, 2003; pp. 119-194.

74. Yang, C.; Xu, P.; Xie, S.; Yao, S. Mechanical performances of four lattice materials guided by topology optimization. Scr. Mater. 2020, 178, 339-345. [CrossRef]

75. Ashby, M.F.; Bréchet, Y.J.M. Designing hybrid materials. Acta Mater. 2003, 51, 5801-5821. [CrossRef]

76. Greer, J.R.; Deshpande, V.S. Three-dimensional architected materials and structures: Design, fabrication, and mechanical behavior. MRS Bull. 2019, 44, 750-757. [CrossRef] 